REVIEW • OPEN ACCESS

\section{Femtosecond optical parametric oscillator frequency combs}

To cite this article: Yohei Kobayashi et al 2015 J. Opt. 17094010

View the article online for updates and enhancements.
Related content

\author{
Advances in ultrafast optical parametric \\ oscillators \\ D T Reid, J Sun, T P Lamour et al. \\ Roadmap on ultrafast optics \\ Derryck T Reid, Christoph M Heyl, Robert \\ $\mathrm{R}$ Thomson et al. \\ - Topical Review \\ P Maddaloni, P Cancio and P De Natale
}

\section{Recent citations}

- A decade of astrocombs: recent advances
$\frac{\text { in frequency combs for astronomy [Invited] }}{\text { Richard A. McCracken et al }}$
- Femtosecond OPO based on MgO:PPLN
$\frac{\text { synchronously pumped by a } 532 \mathrm{~nm} \text { fiber }}{\text { laser }}$
Jianjun Cao et al
$\frac{\text { Mid-infrared optical parametric oscillators }}{\text { and frequency combs for molecular }}$
$\frac{\text { spectroscopy }}{\text { M. Vainio and L. Halonen }}$




\title{
Review
}

\section{Femtosecond optical parametric oscillator frequency combs}

\author{
Yohei Kobayashi ${ }^{1}$, Kenji Torizuka ${ }^{2}$, Alireza Marandi ${ }^{3}$, Robert L Byer ${ }^{3}$, \\ Richard A McCracken ${ }^{4}$, Zhaowei Zhang ${ }^{4}$ and Derryck T Reid ${ }^{4}$ \\ ${ }^{1}$ The Institute of Solid State Physics, The University of Tokyo, 5-1-5 Kashiwanoha, Kashiwa 277-8581, \\ Japan \\ ${ }^{2}$ National Institute of Advanced Industrial Science and Technology, 1-1-1 Umezono, Tsukuba 305-8568, \\ Japan \\ ${ }^{3}$ E.L. Ginzton Laboratory, Stanford University, Stanford CA, 94305, USA \\ ${ }^{4}$ Scottish Universities Physics Alliance (SUPA), Institute of Photonics and Quantum Sciences, School of \\ Engineering and Physical Sciences, Heriot-Watt University, Riccarton, Edinburgh EH14 4AS, UK \\ E-mail: r.a.mccracken@hw.ac.uk
}

Received 12 March 2015, revised 20 May 2015

Accepted for publication 18 June 2015

Published 3 September 2015

\begin{abstract}
Techniques to measure and manipulate the carrier-envelope phase within femtosecond optical parametric oscillators (OPOs) allow their outputs to be stabilized in a way that produces a frequency comb structure, potentially tunable throughout the transparency band of the gain material. In this review we describe the fundamental principles of phase control, on which the development of singly- and doubly-resonant OPO frequency combs is based. We give examples of practical embodiments of such combs, and discuss in detail several applications, including spectroscopy, metrology, quantum computation and astrophotonics.
\end{abstract}

Keywords: frequency combs, optical parametric oscillators, femtosecond lasers

\section{Introduction}

Second-order nonlinear frequency conversion offers a means of extending frequency comb technology to wavelength regions not addressable directly by common femtosecond laser comb sources such as Ti:sapphire [1], Yb:fibre [2], Er: fibre [3], Tm:fibre [4], Cr:ZnS [5], or Cr:ZnSe [6] lasers. Single-pass difference frequency generation (DFG) with femtosecond pulses makes it possible to produce combs having a stable carrier-envelope phase (CEP) [7], even allowing high conversion efficiency to be achieved when using amplifiers in both pumping channels [8]. Constructing a resonator around such a DFG crystal so that oscillation is established from parametric fluorescence with only a single pump laser and by synchronous feedback of the signal and / or idler pulses was first demonstrated in the femtosecond regime in 1989 [9], laying the groundwork for the synchronously pumped optical parametric oscillator (OPO) systems described in this article.
Femtosecond OPOs are natural systems for generating frequency combs, whose applications tend to emphasize the importance of bandwidth and wavelength coverage, for example their use in optical metrology [10], spectroscopy [11], optical clockworks [12] or for comparisons between different time and frequency standards [13]. Unlike laser media, the wavelengths generated in a parametric frequency conversion process are not limited by a prescribed set of electronic energy levels, allowing OPOs to be both widely tunable and broadband, only limited by the absorption characteristics of the nonlinear gain material.

The parametric gain process is also intrinsically quiet. While in a femtosecond laser, contributions from spontaneous emission can increase the noise power spectral density (PSD) $[14,15]$, in an OPO the gain process is instantaneous and the contribution from parametric fluorescence is typically negligible. Furthermore, the synchronously pumped nature of femtosecond OPOs means that they do not need to utilize significant levels of Kerr nonlinearity in order to support 


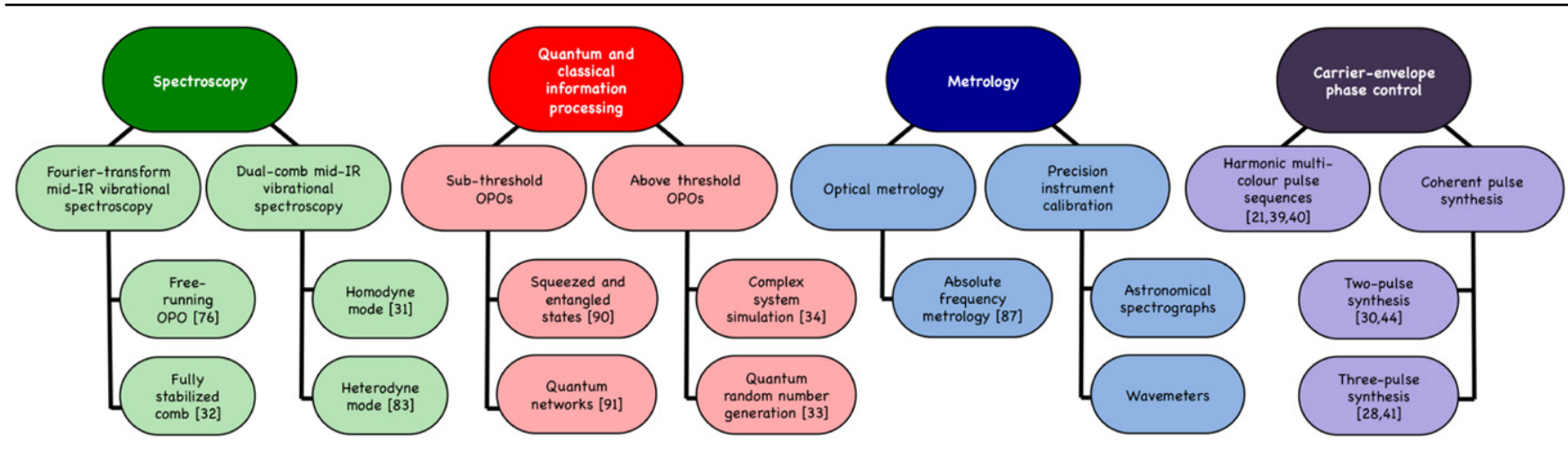

Figure 1. A perspective on femtosecond OPO frequency combs, classifying their main application areas.

modelocking, in contrast to many passively modelocked lasers. Femtosecond OPOs typically employ millimetrelength nonlinear crystals, which are orders of magnitude shorter than the gain media used in fibre frequency combs operating at similar near-infrared wavelengths. This means that the coupling of intensity fluctuations into phase noise within an OPO comb can be much lower than in fibre combs. When a high quality pump laser is used, this feature allows OPO frequency combs to be stabilized to operate with carrierenvelope-offset (CEO) frequency beat linewidths of a few $\mathrm{Hz}$ [16], rather than the hundreds of $\mathrm{kHz}$ associated with competitive laser combs operating in the same spectral region such as Er:fibre [3] and Cr:forsterite [17], although we note that with suitable techniques the offset-frequency linewidth in Er:fibre combs can been reduced to $\mathrm{Hz}$ levels [18]. As well as the differences in the intrinsic noise processes between OPOs and lasers, this difference in performance can also be attributed substantially to the presence of phase noise at the extremes of the super-continuum used for detecting the comboffset beat frequency with the $f$-to- $2 f$ technique [19], which may be lower in an OPO comb which can use more central super-continuum wavelengths for locking, depending on the specific wavelengths of interest [20].

Optical parametric down-conversion is further characterized by the mutual coherence between the interacting fields, studies of which paved the way to the original demonstration of femtosecond OPO frequency combs in the form of relative phase measurements between OPO harmonic outputs [21] and the energy conservation in the parametric process [22]. This phase coherence now plays an important role in the techniques used to stabilize the offsets of OPO frequency combs [23] and in the interpretation and design of degenerate OPO frequency combs [24].

Since their first demonstration in 2007 [20], femtosecond OPO combs have been subject to increasing development. Notable results include the demonstration of average powers exceeding $1 \mathrm{~W}$ [25], zero-offset combs offering phase coherence among all the parametrically related fields [26], $\mathrm{GHz}$ operation [27], tunability into the visible [28] and midinfrared [25], pulses as short as five cycles [29], Hz-level offset-frequency linewidths [16] and as low as 30 attosecond timing jitter between pulses in the same parametrically related comb [30]. Figure 1 presents an overview of the scientific areas already impacted by femtosecond OPO frequency combs. In some of these, the OPO comb offers a technical advantage because alternative laser technology does not yet exist (e.g. in mid-IR vibrational spectroscopy, for which solid-state femtosecond laser sources operating at wavelengths $>2.5 \mu \mathrm{m}$ do not yet exist). In other areas the femtosecond OPO comb is fundamentally unique, for example in quantum optical applications which exploit the properties of parametric entanglement.

Before going further it is necessary to define what we mean by a 'frequency comb'. Formally, a frequency comb is an ensemble of equally spaced narrow frequencies, whose relative phases are constant and whose absolute positions in frequency are fixed. By 'narrow' we mean that the linewidth of an individual frequency is much narrower than the spacing between adjacent frequencies. A modelocked laser naturally satisfies the requirement for fixed relative phases and equal frequency spacing, but freezing the absolute positions of these frequencies is only achieved in practice with the additional step of CEO frequency stabilization, often requiring considerable additional technical effort. While not every example we describe here demands full CEO stabilization, the majority require the offset frequency to have sufficient passive stability for the comb to be static in frequency during the lifetime of the measurement, for example in dual-comb OPO spectroscopy. Others (e.g. coherent synthesis) require that the relative offsets of different combs are controlled.

The remainder of this article develops the core concepts underpinning femtosecond OPO combs, starting from an understanding of the fundamental phase relationships between the pump, signal and idler pulses in a synchronously pumped femtosecond OPO. We highlight the recent achievements in this research area, which can now be divided into singlyresonant and doubly-resonant OPOs (DROs), which are each characterized by a distinct set of performance features and technical approaches. These separate comb configurations are explained in detail, allowing their relative performance to be critically compared. As the technology matures, the uses of femtosecond OPO combs are becoming increasingly diverse and ambitious. Applications employing some or all of the phase-stability characteristics of femtosecond OPOs are discussed in detail and include dual-comb spectroscopy [31], Fourier-transform spectroscopy [32], photonic random number generation [33], coherent pulse synthesis [30], quantum simulation [34] and even the generation of entangled states [35]. 

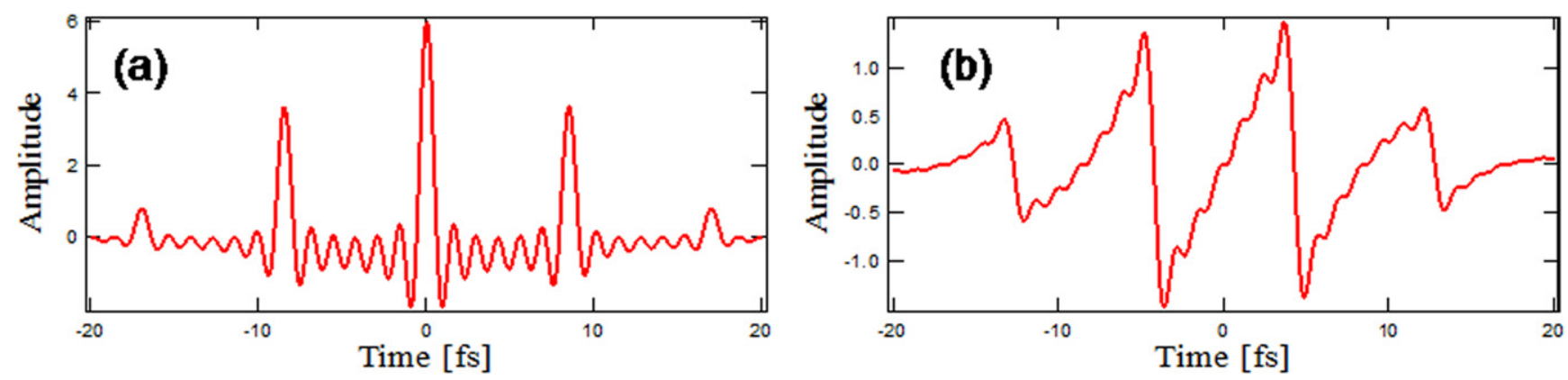

Figure 2. Example illustrating the electric field synthesis of six harmonics pulses to form an attosecond pulse train (a) and a saw-tooth wave (b) with wavelengths of $2550,1275,850,638,510$, and $425 \mathrm{~nm}$.

\section{CEP control in femtosecond OPO combs}

In an optical parametric process, where a pump beam produces a signal and an idler beam, for both the continuous and pulsed modes of operation the optical phase relationship between all three waves is given (modulo $2 \pi$ ) by

$$
\theta_{p}=\theta_{s}+\theta_{i}+\frac{\pi}{2}
$$

where $\theta_{p}, \theta_{s}$, and $\theta_{i}$ are the phases of the pump, signal, and idler, respectively. In a doubly-resonant OPO it is well known that, as a result of the above-mentioned phase relationship, the oscillation is sensitive to the cavity length [36]. When we consider the phase relationship in a singly-resonant OPO (SRO) pumped by a mode-locked pulse sequence, the pulse repetition frequencies of both the pump and signal need to be the same. Here, the optical phase of the signal is determined from the cavity length and the intracavity dispersion, and that of the idler by the phase relation in equation (1). This assumption was experimentally verified in 2000 [21]. The optical phase of a femtosecond OPO can also refer to the $\mathrm{CEP}$, which is defined as the phase difference of the carrier wave relative to the pulse envelope.

In the frequency domain, a femtosecond pulse sequence is represented by a series of equally spaced spectral lines and forms a frequency comb. The position of each comb tooth exhibits a CEO frequency which corresponds to the CEP slip of successive pulses. The optical frequencies of individual comb teeth in the pump, $f_{p}$, signal, $f_{s}$, and idler, $f_{i}$, beams can be expressed as

$$
\begin{aligned}
& f_{p}=f_{0 p}+l f_{\text {rep }}, \\
& f_{s}=f_{0 s}+m f_{\text {rep }}, \\
& f_{i}=f_{0 i}+n f_{\text {rep }},
\end{aligned}
$$

where $l, m, n$ are integers, $f_{\text {rep }}$ is the pulse repetition frequency and $f_{0 p}, f_{0 s}$, and $f_{0 i}$ are the CEO frequencies of the pump, signal and idler beams, respectively. Since the three optical fields also satisfy the simple equation of energy conservation, $f_{p}=f_{s}+f_{i}$, the CEO frequency relation can be expressed as

$$
f_{0 p}=f_{0 s}+f_{0 i} \text {. }
$$

For a given wave, $j$, the CEO frequency and the CEP slip of successive pulses $\left(\Delta \theta_{j}\right)$ are tied together by a simple relation

$$
f_{0 j}=\frac{\Delta \theta_{j}}{2 \pi} f_{\text {rep }}
$$

From equation (1), the CEP slip relation between the pump, the signal, and the idler then follows

$$
\Delta \theta_{p}=\Delta \theta_{s}+\Delta \theta_{i}
$$

Thus, controlling the relative $\mathrm{CEO}$ frequencies in equation (3) is equivalent to controlling the relative CEP slips of equation (5). However, as three fields are involved in the generation of a frequency comb in an OPO, to establish a fixed CEP frequency relationship between all three requires the absolute phase difference between them to be controlled.

By controlling the relative phase relation it is possible to realize numerous applications in various research areas. One promising application is the coherent synthesis of femtosecond pulses as originally envisioned by Hänsch and Shimoda $[37,38]$. The artificial coherent addition of different coloured pulses enables arbitrary waveform generation to be achieved to produce, for example, attosecond pulse trains or saw-tooth waves, which could be applied to the coherent control of chemical reactions. An illustration of coherent synthesis is shown in figure 2, which depicts theoretical results achieved by combining six harmonic pulses to generate specific target waveforms.

For coherent synthesis, it can be beneficial to set the optical frequency ratio between the pump, the signal, and the idler to $3: 2: 1$. Such a sub-harmonic OPO is not only relevant to applications such as arbitrary waveform generation, but also facilitates the direct observation and the control of the optical phase relation. Figure 3 illustrates how the relative CEP slip in the sub-harmonic femtosecond OPO can be measured and controlled [39].

Here, we consider a sub-harmonic OPO pumped by a Ti:sapphire laser at a centre wavelength of $850 \mathrm{~nm}$. The pump, the signal, and the idler frequencies are defined as $3 \omega$, $2 \omega$, and $\omega$, respectively. The generation of these harmonics is readily available by exploiting the additional frequency conversion processes that occur when using a periodically-poled lithium niobate (PPLN) crystal as the nonlinear medium in the OPO. Interactions between the pump, signal and idler pulses 


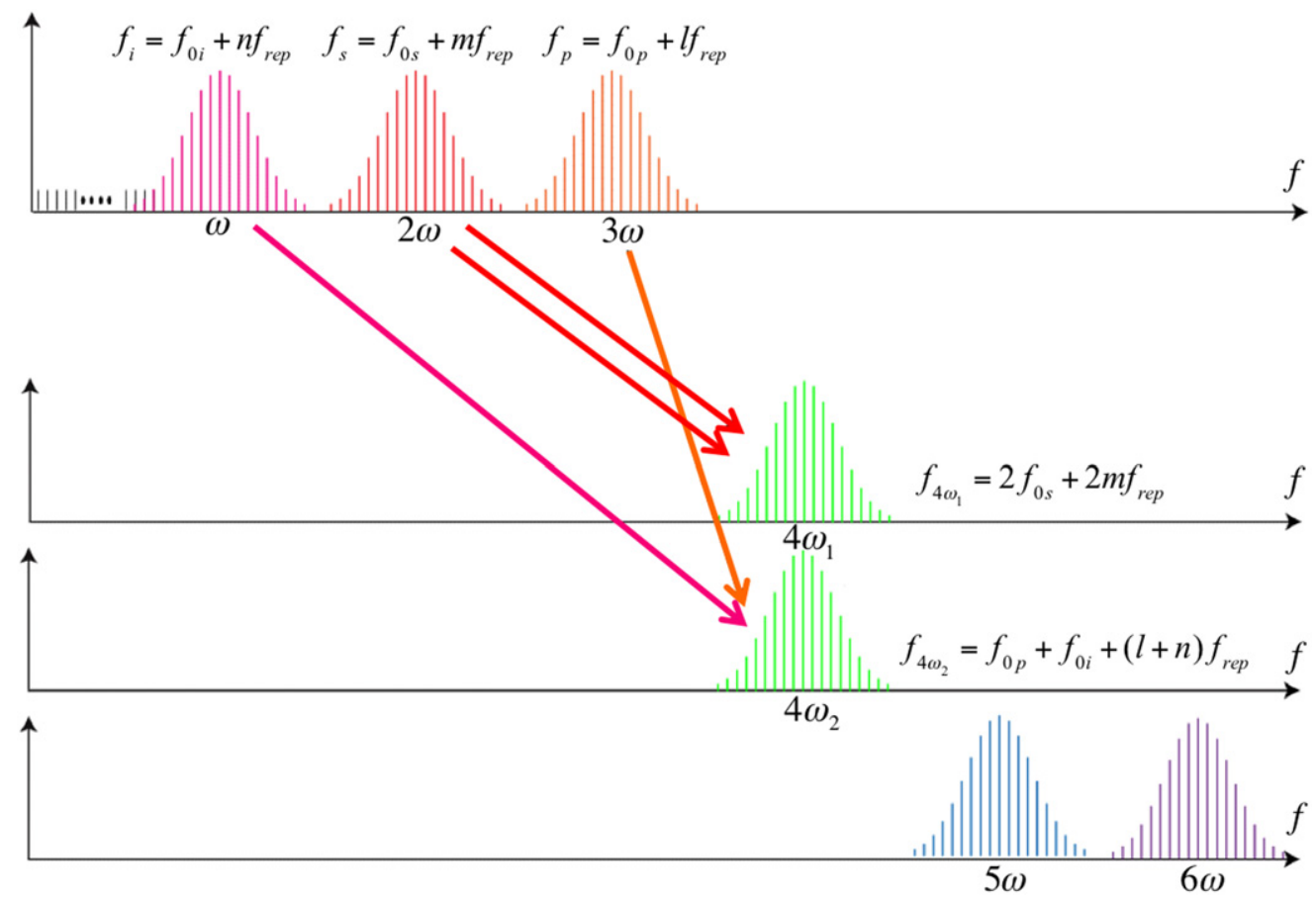

Figure 3. Relative CEP relations between sub-harmonics in a 3:2:1 OPO. The pump ( $3 \omega)$, signal $(2 \omega)$ and idler $(\omega)$ combs can mix either by sum-frequency generation or second-harmonic generation to form two new combs around the same frequency $\left(4 \omega_{1}\right.$ and $\left.4 \omega_{2}\right)$, whose heterodyne beat reveals the relative offsets between the pump, signal and idler combs. The same approach can be extended to higher harmonics $(5 \omega$ and $6 \omega)$. See text for full details.

produce new pulse trains due to sum-frequency mixing (SFM) and second-harmonic generation (SHG). The new corresponding frequency combs can be described by

$$
f_{\mathrm{SFM} / \mathrm{SHG}}=q f_{0 p}+r f_{0 s}+s f_{\text {rep }},
$$

with the idler CEP not being explicitly shown as it can be eliminated using equation (3). Along with the generation of the signal and the idler, the second harmonic of the signal $\left(4 \omega_{1}\right)$ and the sum frequency between the pump and the idler $\left(4 \omega_{2}\right)$ are produced and their optical frequencies can be set to be equal. The resulting beat frequency $\left(f_{\text {beat }}\right)$ from the two generated $4 \omega$ signals can be expressed as:

$$
f_{\text {beat }}=2 f_{0 s}-\left(f_{0 p}+f_{0 i}\right)=3 f_{0 s}-2 f_{0 p}=f_{0 s}-2 f_{0 i} .
$$

If the beat frequency is zero, i.e. the offset frequencies have a ratio of $f_{0 i}: f_{0 s}: f_{0 p}=1: 2: 3$, then according to equations (3) and (5), the CEP slips follow the same ratio $\Delta \theta_{i}: \Delta \theta_{s}: \Delta \theta_{p}=1: 2: 3$. Since the wavelength ratio between the pump, the signal, and the idler, in this order, is $1: 2: 3$, the CEP slip incurred during successive pulses remains the same in the time domain. In this case, the synthesized electric field shape does not change from pulse-to-pulse. Only the position of the synthesized field relative to the synthesized pulse envelope shifts, in accordance to the absolute value of $f_{0 p}$. The sum frequency between the pump and the signal $(5 \omega)$, and the second harmonic of the pump $(6 \omega)$, are also generated. The femtosecond PPLN OPO pumped by a Ti:sapphire laser thus generates seven differently coloured pulses simultaneously. The offset frequency relation between the pump and the signal can be controlled by tuning the cavity length. Slight changes to the cavity length during laser oscillation do not affect the repetition rate. Only the carrier frequency shifts to compensate the intracavity dispersion to realize the same repetition frequency as the pump. Under these conditions the slight cavity-length change possible by using a piezoelectric transducer (PZT) causes the phase relation to change in the OPO.

This method for observing and controlling the phase relation was first proposed and demonstrated in the early 2000s [40, 41]. Around the same time, the observation and the control of the CEO frequency in Ti:sapphire lasers was achieved, which led to the realization of the optical frequency comb and CEP control in oscillators [42, 43]. For coherent synthesis, establishing a phase relation of $\Delta \theta_{i}: \Delta \theta_{s}: \Delta \theta_{p}=1: 2: 3$ fixes both the relative phase relation in the time domain and the synthesized electric field shape shift with respect to the phase slip of the idler. If the relative phase is fixed to $\Delta \theta_{s}=\Delta \theta_{p}$ then the pump and the signal have the same offset frequency, which implies that they can be synthesized as a single pulse. CEP control and subsequent superposition of two-colour pulses in an OPO makes it possible to realize a synthesized envelope shape which is shorter than those of the original pulses [44]. The combination of relative phase measurements with the absolute phase measurement of the pump makes it possible to fix all the parameters. By using a similar technique, one can superimpose independent laser pulses with different wavelengths coherently for field synthesis. For example, the phase control of a Ti:sapphire laser and another type of mode-locked laser with different colour can 


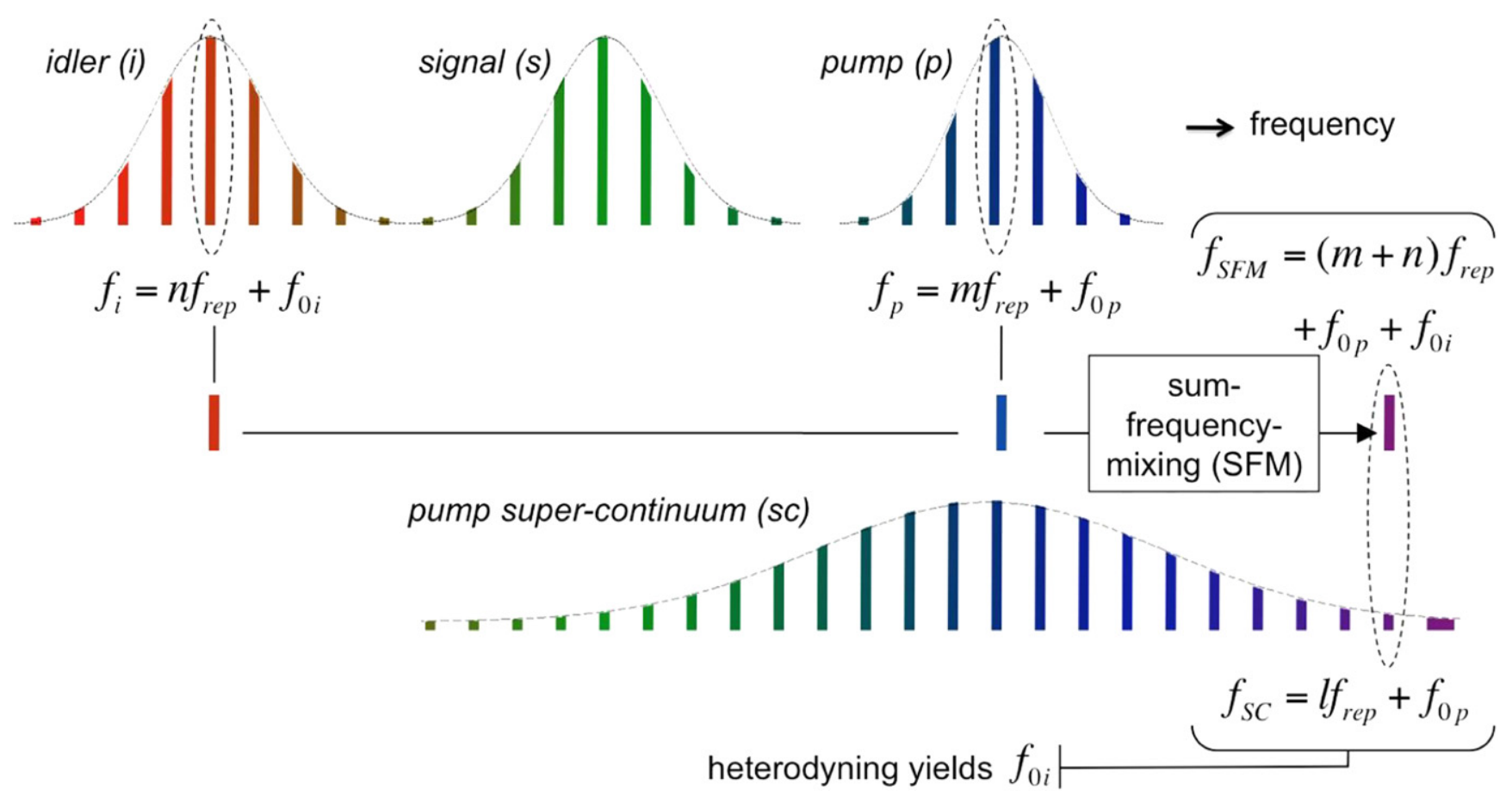

Figure 4. Illustration of the measurement of the idler-comb carrier-envelope offset frequency, $f_{0 i}$. Sum-frequency mixing between the pump $(p)$ and idler $(i)$ combs yields a secondary comb with an offset of $f_{0 p}+f_{0 i}$. Heterodyning this comb with pump super-continuum light of the same wavelength (comb offset $f_{0 p}$ ) generates a beat frequency which eliminates the pump comb offset leaving only frequencies at the harmonics of the repetition rate and $\pm f_{0 i}$.

lead to the realization of an ultra-broadband spectrum for ultrashort pulse generation [45-49]. Direct frequency conversion from a long-wavelength laser is another possible candidate for producing phase-coherent multi-colour pulses. Recently, Cr-doped crystals have been shown to be suitable candidates for the production of mid-infrared femtosecond pulses [5, 6]. Tm-doped fibres [4] are also promising possibilities, since the pulses can be amplified to high average powers.

Ultimately, a difficulty still remains in the direct measurement of the shape of the synthesized electric field when using the low-energy pulses available from OPOs. The superposition of parallel optical parametric amplifiers makes it possible to increase the peak power to estimate the absolute phase relation between different colour pulses. Recently, multi-colour OPAs were developed for high-intensity coherent synthesis at $1 \mathrm{kHz}$ repetition frequency [48, 49] and higher repetition-rate OPAs have also been demonstrated [50].

\section{Single-resonant femtosecond OPO frequency combs}

In a SRO, the CEO frequency of the signal (or idler) pulses can be measured by using interference between a pump supercontinuum and the pump + signal (or pump + idler) SFM light. As an example, figure 4 illustrates how the heterodyne beat between the pump super-continuum comb, $f_{\mathrm{SC}}=l f_{\text {rep }}+f_{0 p}$, and the comb corresponding to the pump + idler SFM light, $f_{\mathrm{SFM}}=(m+n) f_{\text {rep }}+f_{0 p}+f_{0 i}$, contains the idler carrier-envelope offset frequency $f_{0 i}$, and harmonics of the laser repetition frequency, $f_{\text {rep }}$. By detecting this heterodyne beat experimentally and locking it to a stable frequency reference it is possible to stabilize the carrier-envelope offset frequency of an SRO femtosecond idler frequency comb. An equivalent approach can also be taken to stabilize the signal comb by beating pump + signal sum-frequency light with the pump super-continuum. In either case, with the additional constraint of stabilizing the pump laser repetition frequency, a fully stabilized frequency comb can be realized, in which the frequencies of every optical mode can be precisely known.

SRO frequency combs were first demonstrated in 2007 by Sun et al [20], in which a composite frequency comb spanning $0.4-2.4 \mu \mathrm{m}$ was achieved by phase locking a femtosecond Ti:sapphire laser and an OPO to a common supercontinuum reference. A custom-built Kerr-lens modelocked Ti:sapphire laser (200 MHz, $800 \mathrm{~nm}, 1.3 \mathrm{~W}, 50 \mathrm{fs})$ was used to synchronously-pump an OPO based on a $1 \mathrm{~mm}$ long MgO:PPLN crystal. An end mirror of the pump laser was mounted on a PZT to lock the repetition rate to a stabilized external $200 \mathrm{MHz}$ reference. Approximately 20\% of the pump power was launched into a $30 \mathrm{~cm}$ long photonic crystal fibre (PCF) to obtain an octave-spanning super-continuum for $f$-to- $f$ self-referencing [42]. The detected CEO frequency $f_{0 p}$ was controlled using a feedback loop, with an acousto-optic modulator in the Ti:sapphire pump beam used to modulate the intracavity dispersion of the laser. The remaining $80 \%$ of the pump power was used to pump the OPO, which was resonant from 1.2 to $1.37 \mu \mathrm{m}$. Last-coherence length and higher-order phase-matching in the OPO crystal produced a number of 


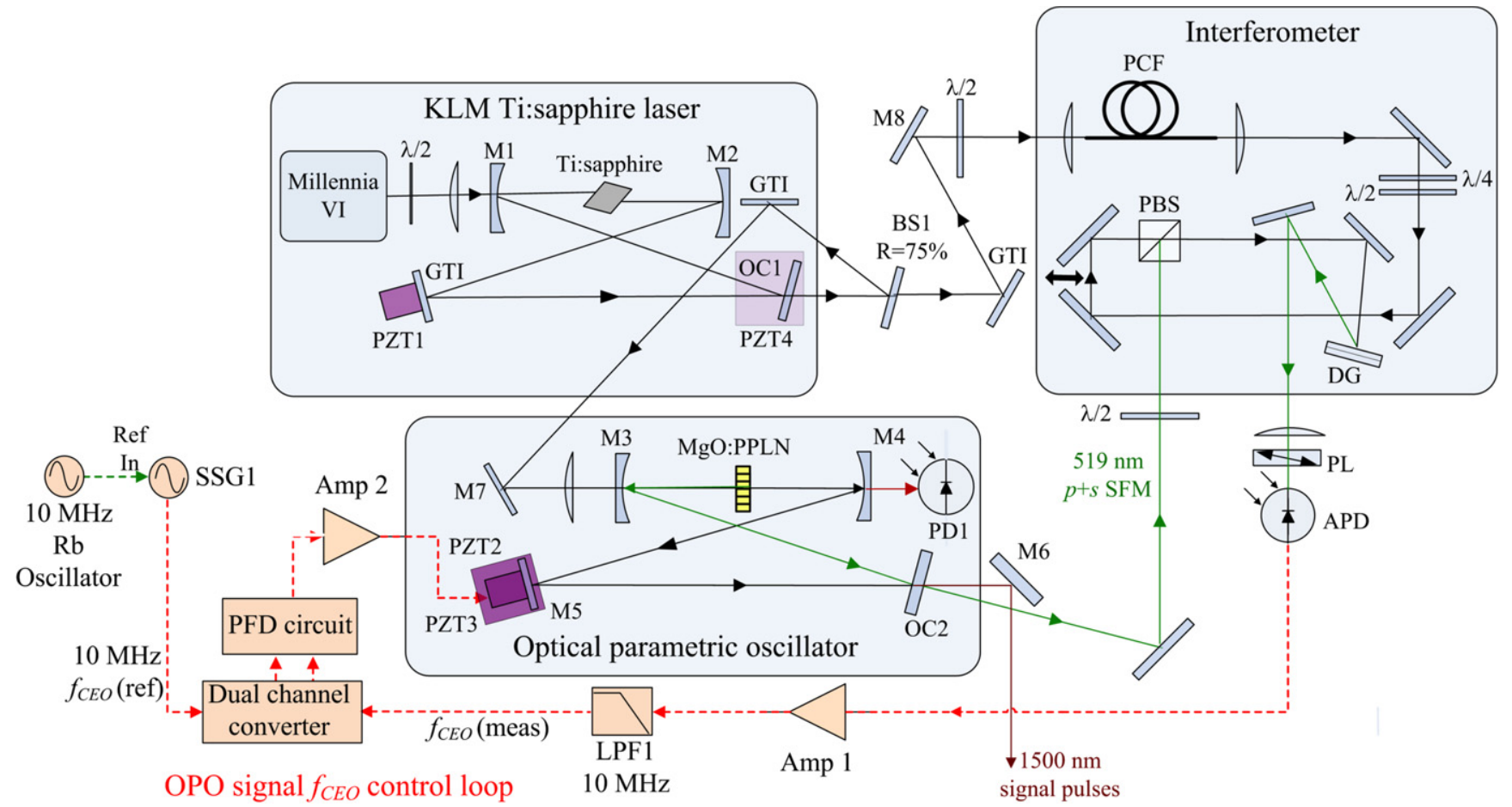

Figure 5. Schematic of the optical and electronic layouts used to generate an OPO frequency comb without $f$-to- $2 f$ self-referencing. Reprinted with permission from [51].

SHG and SFM pulses which, combined with the non-resonant idler, spanned the visible to mid-IR spectral regions.

The SHG signal $(2 s)$ was heterodyned against the pump super-continuum in a nonlinear interferometer to obtain a beat frequency at $2 f_{0 s}-f_{0 p}$, which was used to drive PZT2 in the OPO cavity and complete the phase-locking loops for the pump and $2 s$ pulses, providing full CEO control of all generated frequency combs. A heterodyne frequency between the pump super-continuum and pump + idler (pi) SFM pulses was used to assess the locking quality of the experiment, which was found to stabilize the comb lines to a near-instrumentlimited linewidth of $1.2 \mathrm{kHz}$.

In 2009 Adler et al [25] used an amplified Yb:fibre laser (136 MHz, $1.07 \mu \mathrm{m}, 10 \mathrm{~W}, 100 \mathrm{fs}$ ) to pump an OPO with a $7 \mathrm{~mm}$ long MgO:PPLN crystal. The OPO cavity had high finesse at the resonant signal wavelength, and produced up to $1.5 \mathrm{~W}$ of idler power, tunable from 2.8 to $4.8 \mu \mathrm{m}$. The higher pump power and intracavity signal power generated an increased number of parasitic mixing pulses at the $\mathrm{mW}$-level, including wavelengths as low as $360 \mathrm{~nm}(4 s)$. The pump super-continuum heterodyning method described above was implemented with both $p s$ and $p i$ pulses, with the $f_{0 s}$ beat from the former used to stabilize the OPO cavity length and the latter $f_{0 i}$ beat used to modulate the fibre laser's pumpdiode power, which acts on $f_{0 p}$. After stabilization of the Yb:fibre repetition rate, the OPO comb quality was measured using an out-of-loop $10 \mathrm{kHz}$ linewidth external cavity diode laser. Using this method the idler comb linewidth was found to be $40 \mathrm{kHz}$.

In 2010 Ferreiro et al [23] demonstrated that the CEO frequency of the pump laser does not need to be controlled in order to achieve high-quality stabilization of the OPO signal pulses. A free-running Ti:sapphire laser $(280 \mathrm{MHz}, 793 \mathrm{~nm}$, $1.4 \mathrm{~W}, 50 \mathrm{fs}$ ) was used to pump an OPO based on $1 \mathrm{~mm}$ of MgO:PPLN which was signal resonant over $1.42-1.60 \mu \mathrm{m}$ (figure 5). $350 \mathrm{~mW}$ of pump light was used to generate a super-continuum which was interfered with ps pulses at $520 \mathrm{~nm}$. The resulting beat provided the CEO frequency of the signal pulses, and was controlled through a PZT mounted on an OPO cavity mirror. The locked beat had an instrumentlimited bandwidth of $1 \mathrm{kHz}$, and phase noise PSD measurements showed a cumulative phase error of $0.38 \mathrm{rad}$ in $1 \mathrm{~s}$, integrated up to $1 \mathrm{MHz}$.

Further investigations of these results [16] were carried out and the cavity length of the Ti:sapphire laser stabilized to the 8th harmonic of the repetition rate $(2.24 \mathrm{GHz})$, referenced to a synthesized signal generator. Both the signal generator and the $f_{0 s}$ beat were referenced to the same $10 \mathrm{MHz}$ Rb clock. Locking the pump repetition rate reduced the linewidth of the offset frequency beat, $f_{0 s}$, which was measured to be $15 \mathrm{~Hz}$ at the $-3 \mathrm{~dB}$ point with an instrument resolution of $10 \mathrm{~Hz}$. The cumulative phase noise in this system was measured to be 0.56 rads in $1 \mathrm{~s}$, integrated over $2 \mathrm{MHz}$. Two-sample frequency deviation measurements [52] of the comb instability implied fractional stabilities of $2 \times 10^{-11}\left(f_{\text {rep }}\right)$ and $1 \times 10^{-15}\left(f_{0 s}\right)$, comparable with the $2 \times 10^{-11}$ instability in the Rb clock.

At Heriot-Watt University we have recently demonstrated a set of fully stabilized frequency combs from a $1 \mathrm{GHz}$ fundamentally-pumped OPO that spanned the visible and IR. CEO frequency stabilization of the Ti:sapphire laser (Gigajet, Laser Quantum) was achieved in a standard $f$-to- $2 f$ interferometer, with $f_{0 p}$ controlled via diode-current modulation in 


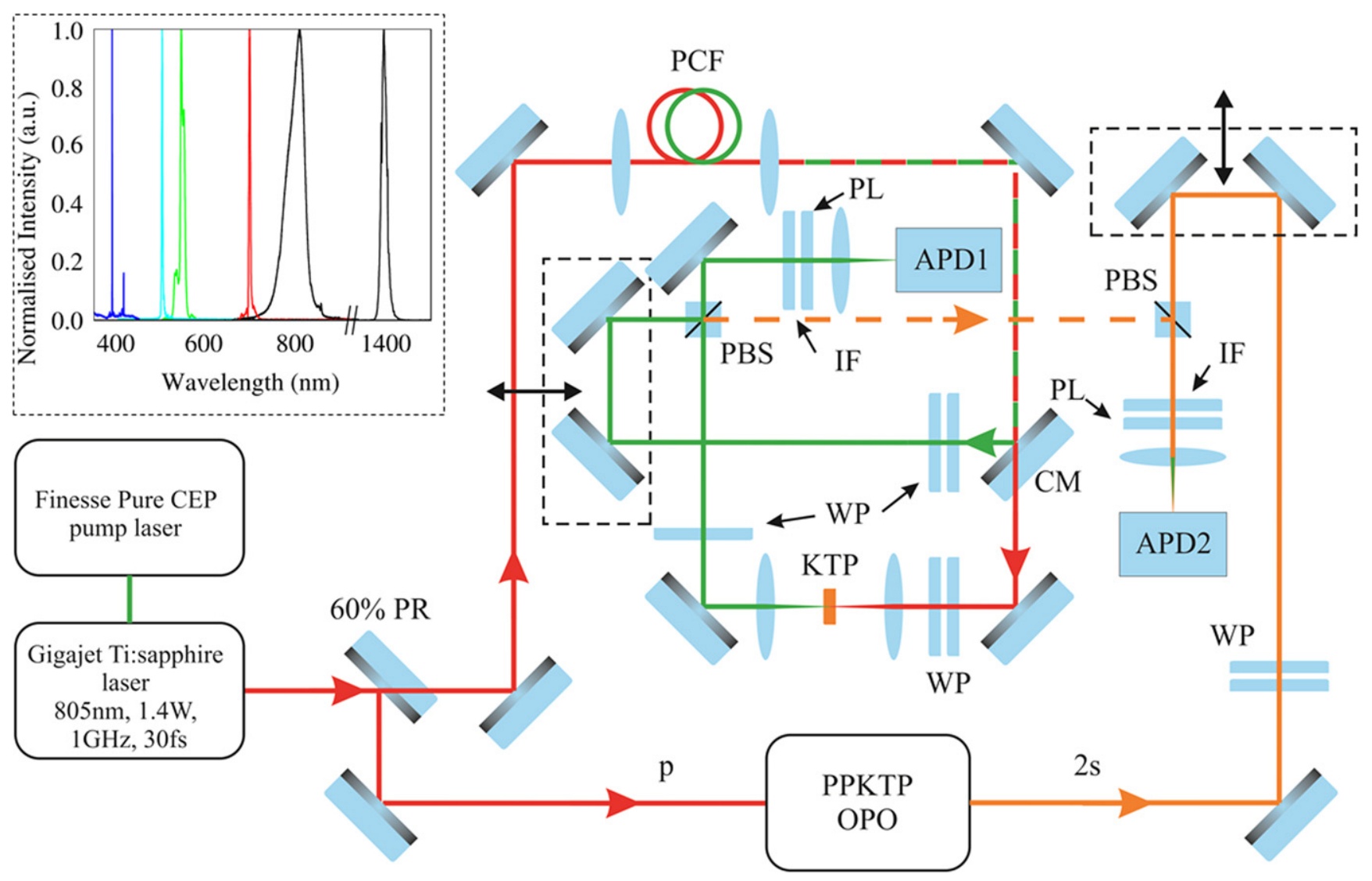

Figure 6. Optical layout of the scheme used simultaneously lock all OPO frequency combs. APD, avalanche photodiode; CM, cold mirror; IF, interference filter; PBS, polarizing beam splitter; PCF, photonic crystal fibre; PL, polarizer; PR, partial reflector; WP, wave plate. Inset: spectra of the composite comb comprising SHG pump (400 nm); pump + signal (510 nm) and pump + idler (565 nm) SFM light; SHG signal $(700 \mathrm{~nm})$; pump $(805 \mathrm{~nm})$; and signal $(1400 \mathrm{~nm})$. The idler spectrum $(1900 \mathrm{~nm})$ is not shown.

the pump laser (Finesse Pure CEP, Laser Quantum). In a complementary arrangement, $2 s$ pulses were heterodyned against a different portion of the pump super-continuum to obtain a beat that was then used to lock the difference between the pump and signal CEO frequencies. Both CEO frequencies were locked to a $10 \mathrm{MHz}$ Rb clock, which also acted as the frequency reference for the repetition-rate stabilization of the Ti:sapphire laser. The optical layout is shown in figure 6, with the spectra of the composite comb inset. Controlling both the Ti:sapphire laser and the OPO frequency combs automatically stabilizes all other combs generated on the bench, as is evident from equation (6).

PSD measurements of the Ti:sapphire locking loop showed a cumulative phase noise of 0.4 rad over $64 \mathrm{kHz}$, with the dominant noise at 6 and $33 \mathrm{kHz}$ attributable to RIN fluctuations of the DPSS pump laser. The cumulative phase noise for the OPO was $1.6 \mathrm{rad}$ over $64 \mathrm{kHz}$, with stability limited by the corner frequency of the PZT used for cavity length control. The high repetition rate of the Ti:sapphire laser resulted in low peak power pulses, requiring significant levels of average power $(>500 \mathrm{~mW})$ to generate a coherent octave spanning super-continuum for $f$-to- $2 f$ locking. The accompanying decrease in the available average power for the OPO restricted its operational range to $1375-1425 \mathrm{~nm}$.
Partially-stabilized SRO combs have also been demonstrated in a number of forms. Kobayashi et al achieved phase locking among various harmonically related outputs of an OPO operating in a 3:2:1 pump:signal:idler frequency ratio $[39,40]$. Sun et al extended this to an OPO with no fundamental wavelength constraints by exploiting dual-colour operation, which is possible when the net cavity group delay of the OPO is identical for two different signal wavelengths [53]. Internal beat frequencies were observed in the visible at wavelengths corresponding to $p s, 2 s$ and $p i$, which could be stabilized for use in coherent pulse synthesis [44].

A multi-octave-spanning phase coherent composite OPO frequency comb was recently reported by McCracken et al [28]. The CEO frequencies of the pump, signal and idler pulses were locked to the same frequency comb grid by using a zero-offset frequency locking technique [26]. Through parametric energy conservation [22], all parasitic SHG and SFM combs generated in the OPO were also constrained to this same frequency grid. The natural synchronisation of the OPO with the pump laser allowed the composite frequency comb to be realized without the need for complex locking electronics between separate oscillators $[54,55]$, making this system an ideal candidate for high-repetition rate coherent pulse synthesis. 
Recently, Balskus et al extended the control of SRO frequency combs to the harmonically-pumped regime [27]. Femtosecond OPOs are typically synchronous with their pump laser, with the cavity length of the former matched to the latter. In harmonic pumping the OPO cavity length is chosen to be an integer $[56,57]$ or integer fraction $[58,59]$ of the pump cavity length, allowing high repetition rate pulse trains to be generated without the need for high repetition rate oscillator. Balskus et al used a $333 \mathrm{MHz}$ Ti:sapphire laser to pump an OPO constructed in a fundamental $(333 \mathrm{MHz})$ and harmonic $(1 \mathrm{GHz})$ configuration, locking the CEO frequency of the signal in both cases, and compared the locking stability. The fractional stability of the locked $f_{0 s}$ for both OPOs was $1.35 \times 10^{-15}$ over a $1 \mathrm{~s}$ gate time, demonstrating that harmonic pumping does not reduce the comb quality despite the signal pulses making multiple trips round the cavity, which might be expected to increase environmental noise contributions. It should be noted that while the harmonic OPO comb itself is stable, the underlying comb structure will contain additional sidebands at multiples of the pump repetition rate.

Progress in SRO frequency combs has been encouraging, with instabilities in the positions of the comb lines limited only by the clocks used as a locking reference. Phase instabilities below $200 \mathrm{mrad}$ are readily achievable, making OPO frequency combs competitive with the best laser combs operating in the near-infrared region. Future developments will undoubtedly include extensions to longer wavelength regions which are ideal for spectroscopy and other sensing applications, as well as pushing the boundaries of fundamental pumping using high repetition rate oscillators [60].

\section{Doubly-resonant femtosecond OPO frequency combs}

In DROs the signal and idler waves resonate in the OPO, and the simultaneous cavity field enhancement for the signal and idler can lead to low oscillation thresholds. The threshold of a DRO can be lower than an SRO with the same gain by a factor of half the round-trip power loss [61]. This feature of DROs reduces the gain requirement and consequently compact, low-power, and quiet pump sources can be used. Moreover, shorter nonlinear crystals can be used, which usually results in broad phase matching bandwidths.

The frequencies of the signal and idler must satisfy two criteria: the energy conservation condition and the resonance condition. Therefore, unlike SROs, the wavelength tunability is limited. In most cases, the cavity is shared between the signal and idler, and scanning the cavity length results in discrete oscillation peaks, with widths in the order of a tenth of the wavelength. Achieving stable operation for long times usually requires active stabilization, and can be challenging in the presence of environmental noise or frequency noise on the pump, especially if the frequencies of the signal and idler are far apart.

In the frequency comb regime, the pump is a frequency comb, and the signal and idler are expected to be frequency combs as illustrated in figure 7(a). In the non-degenerate operation, the signal and idler are well separated from each other. They can become close to each other to spectrally overlap, which is shown as partly-degenerate operation in figure 7(a). In this condition, the signal and idler can be independent frequency combs with different CEO frequencies resulting in beating in the overlapping portion of the spectrum [62]. It has been shown by numerical simulations and in experiments that the signal and idler can become a single frequency comb, where the beating disappears $[63,64]$. The rich physics of the nonlinear interactions between the combs in the transition from non-degenerate to degenerate operation is yet to be studied comprehensively.

At degeneracy, the signal and idler waves become indistinguishable, and as a consequence, the phase and frequency of the output is automatically locked to the pump. This is an important feature of degenerate femtosecond OPOs because they can offer mid-infrared frequency combs at their output which are intrinsically phase and frequency locked to the near-IR pump frequency comb [65]. When both the repetition and CEO frequencies of the pump laser are stabilized then the entire pump-OPO system becomes a fully locked frequency comb.

In this regime, the optical carrier frequency of the signal pulses is half the carrier frequency of the pump pulses (the opposite of SHG). The relationship between the phases of pump and signal is given, modulo $2 \pi$, by:

$$
\theta_{p}=2 \theta_{s}+\frac{\pi}{2}
$$

where $\theta_{p}$ and $\theta_{s}$ are the optical carrier phases of pump and signal, respectively [65]. The comb lines of the output frequency comb at degeneracy are defined by:

$$
f_{m}=\frac{f_{0 p}}{2}+m f_{\text {rep }},
$$

or

$$
f_{m}=\frac{f_{0 p}}{2}+m f_{\text {rep }}+\frac{f_{\text {rep }}}{2} .
$$

These two frequency states are different by a shift of half the repetition rate, and the OPO operates in one of them, depending on which one is supported by the resonator. Switching from one state to another is achieved by changing the round-trip cavity length by approximately half of the OPO centre wavelength [65].

Identifying the degenerate operation of OPOs in the frequency comb regime, where the above conditions are satisfied, is sometimes challenging. For instance, operation of femtosecond DROs at many times above the oscillation threshold can result in multiple frequency comb outputs, similar to the micro-resonator-based OPOs [66]. Also, in the partly degenerate and non-degenerate OPO (figure 7(a)) independent signal and idler frequency combs can be observed which do not follow the above equations [67]. In general, where the signal and idler cannot be distinguished spectrally, and the OPO is operating at only a few times above 
a

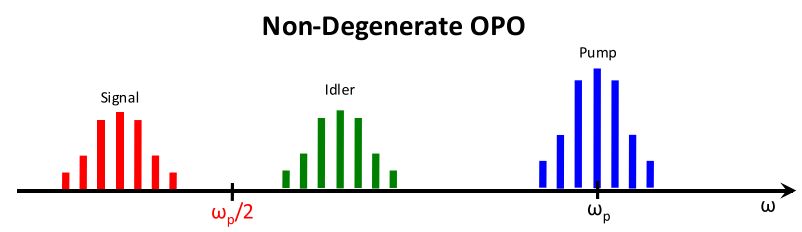

Partly-Degenerate OPO

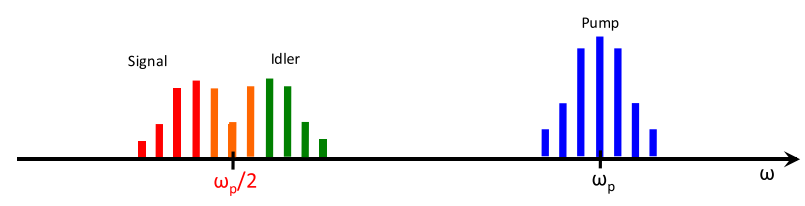

Degenerate OPO

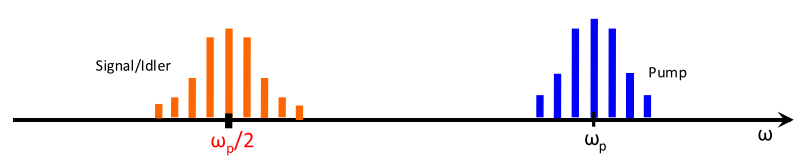

Pump Wavelength $(\mu \mathrm{m})$

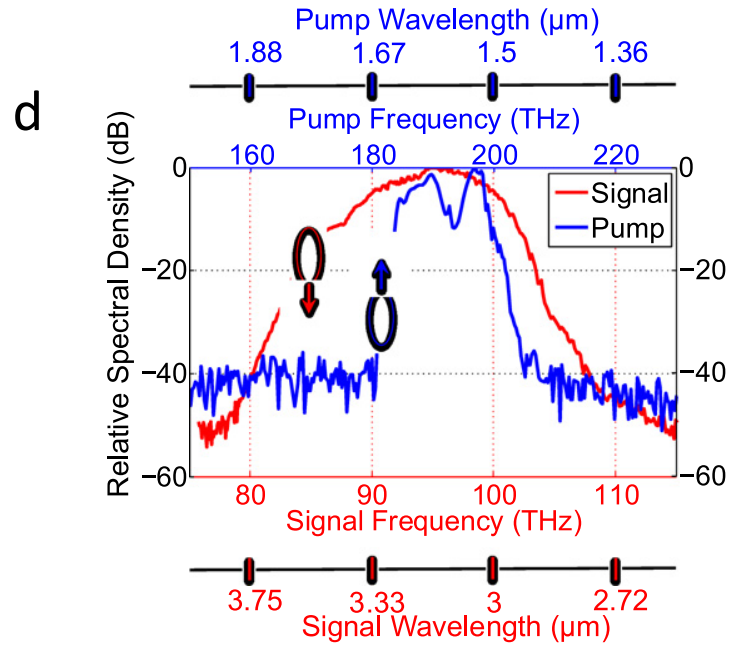

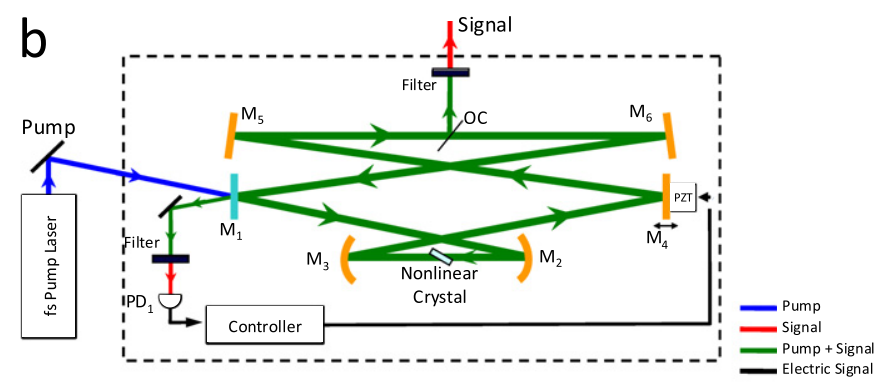

C

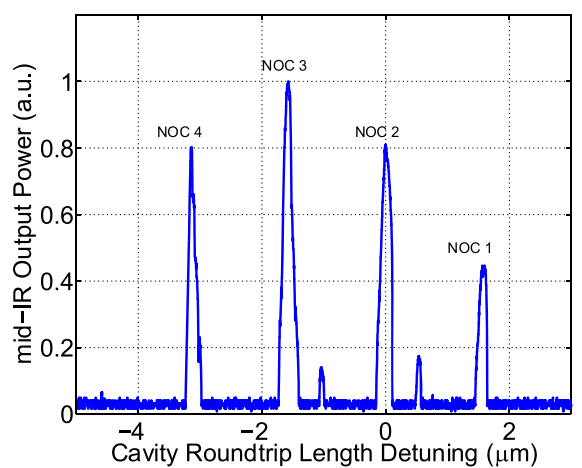

e

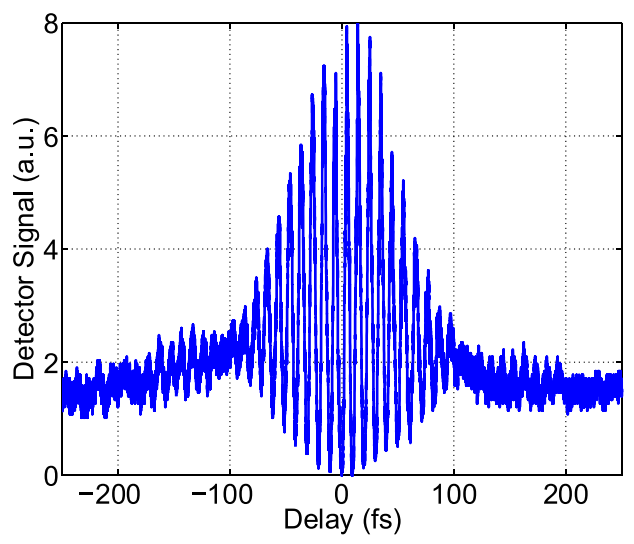

Figure 7. (a) Illustration of the pump, signal and idler frequency combs in non-degenerate, partly degenerate, and degenerate frequency comb OPOs, (b) schematic of a degenerate OPO pumped by a femtosecond pump laser, (c) oscillation peaks of a frequency comb OPO operating around degeneracy, (d) pump and output spectra of a degenerate OPO [65], (e) autocorrelation trace of a degenerate OPO output at $3.1 \mu \mathrm{m}[65]$.

the oscillation threshold, the operation is most likely degenerate with established phase and frequency locking over the spectrum [65].

Several degenerate frequency comb OPOs have been demonstrated over the past few years for extending the wellestablished mode-locked frequency comb sources to longer wavelengths with salient features of low pump power requirement, phase- and frequency- locked operation, and broadband outputs. Different femtosecond pump lasers, such as Ti:sapphire at around $800 \mathrm{~nm}$, mode-locked fibres lasers at around $1 \mu \mathrm{m}$ (Yb-doped), $1.5 \mu \mathrm{m}$ (Er-doped), and $2 \mu \mathrm{m}$ (Tmdoped) have been exploited to generate femtosecond frequency combs at $1.5 \mu \mathrm{m}[68,69], 2 \mu \mathrm{m}$ [70], $3 \mu \mathrm{m}$ [65], and $4 \mu \mathrm{m}$ [29]. Different nonlinear crystals have been used for realization of such OPOs ranging from PPLN $[65,68,70]$ to orientation-patterned GaAs [71] and BiBO [69].

The dominant resonator designs for these OPOs have been the bowtie ring resonator or equivalent folded versions, as depicted in figure 7(b), with a dichroic dielectric mirror (M1) employed for introducing the pump light. The remaining mirrors are usually metal-coated for maximum reflectance in mid-IR. The group velocity dispersion in the cavity is usually compensated to be close to zero and it has been shown that fine-tuning the intracavity dispersion can lead to fewcycle signal pulses in the mid-IR [29]. The crystal length is usually chosen to be short (close to the walk-off length of the pump and signal wavelengths) providing broad gain bandwidth and limiting the dispersion in the cavity. Mode- 

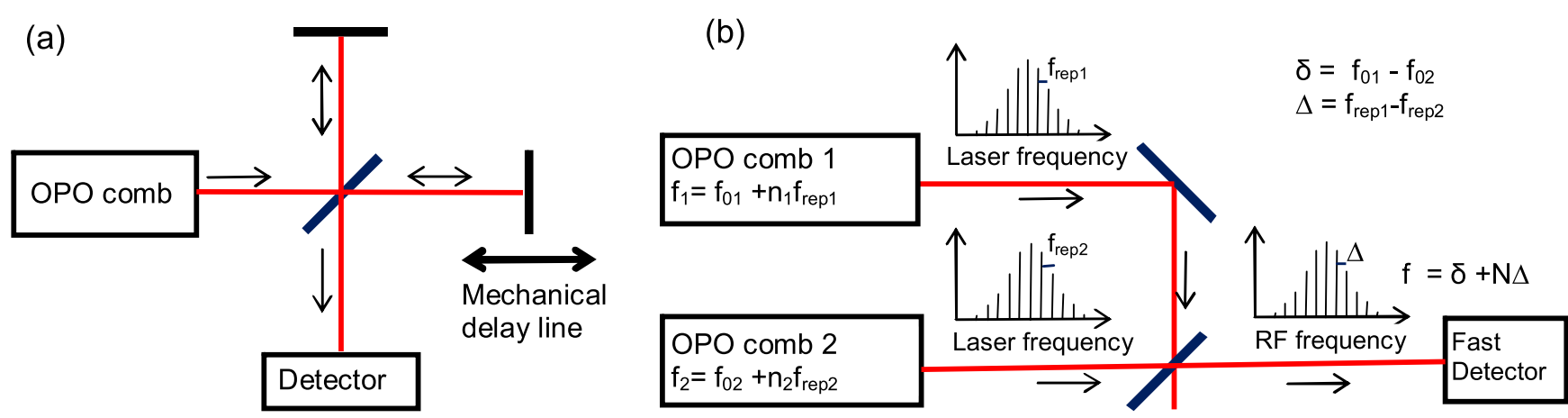

Figure 8. Layout of (a) a single-comb FTIR spectrometer and (b) a dual-comb FTIR spectrometer.

matching lenses are usually employed to achieve optimum pumping.

Similar to synchronously-pumped SROs, the round-trip time of the resonator is matched to the repetition period of the pump, however, because of the phase-sensitive nature of the gain at degeneracy, when the cavity lengths of such OPOs are scanned, multiple oscillation peaks are observed, as depicted in figure $7(\mathrm{c})$. These peaks are usually separated by half the wavelength of the signal, corresponding to the round-trip phase shift steps of $\pi$. Higher-order spatial operation can be observed as extra peaks. For instance, the two weakest peaks of figure 7(c) correspond to operation at $\mathrm{TEM}_{01}$, and the rest are $\mathrm{TEM}_{00}$. Depending on the intracavity dispersion, pump power, and the spectral response of the cavity, different cavity lengths can correspond to non-degenerate, partly-degenerate, and degenerate operation, and the OPO can be locked to a peak for continuous operation. Active stabilization is usually implemented to achieve stable long-term operation lasting for days [65]. In some cases, thermal stabilization has resulted in stable operation over shorter timescales [68].

The dominantly used stabilization method is the top-offringe locking that has been widely used for stabilizing cavities and interferometers. A small length modulation $(\sim 10 \mathrm{~nm}$ in the $\mathrm{kHz}$ range) is applied to the OPO cavity, and the error signal is generated by phase-sensitive detection of the applied modulation frequency in the output signal through a lock-in amplifier or an electronic mixer. The error is then applied to a PZT on the OPO cavity (such as M4 in figure 7(b)) to achieve stable operation at the centre of one of the oscillation peaks [65]. Figure 7(d) shows the pump and output spectrum of a degenerate femtosecond OPO pumped by an Er-doped modelocked fibre laser, and the corresponding autocorrelation trace is depicted in figure 7(e), suggesting shorter than $100 \mathrm{fs}$ pulses centred at $3.1 \mu \mathrm{m}$.

The phase and frequency locking is verified experimentally by achieving stable interference between the output femtosecond pulses of two independent degenerate OPOs pumped by the same pump [65]. It is also verified experimentally that for a free-running mode-locked pump laser, the second-harmonic of the OPO output has a comb linewidth of about $140 \mathrm{kHz}$ which is the same as the comb linewidth of the free-running pump [72]. Preliminary results suggest that this behaviour can be present in cases where sub-Hz linewidths are used for the pump [73], however a comprehensive study of the strength of this intrinsic phase and frequency locking is yet to be undertaken.

In principle, cw OPOs can be very efficient and pump depletions greater than $90 \%$ have been reported. It is expected that the efficiency of half-harmonic generation in cw degenerate OPOs can be as high as $90 \%$ and beyond [74]. So far, a conversion efficiency of slightly higher than $60 \%$ has been achieved for half-harmonic generation of sub-50 fs femtosecond pulses at $2 \mu \mathrm{m}$ [75].

\section{Current and future applications of femtosecond OPO frequency combs}

Stabilized OPO frequency combs, with their exceptional versatility in spectral coverage spanning the visible, nearinfrared and mid-infrared wave bands, present opportunities for innovative applications in frequency metrology, spectroscopy and quantum optics. Here we outline some emerging and potential applications which make use of the unique properties of SRO and DRO frequency combs.

\subsection{Spectroscopy}

The mid-infrared (mid-IR) spectral region is well suited for sensing molecules, since it contains strong characteristic rovibrational transitions of most molecular species. As already discussed, broadband OPOs are readily designed to generate combs in this important spectroscopic region.

Using broadband mid-IR OPO frequency combs, spectroscopy has been conducted with two different schemes. One way is directly using OPO combs as broadband photonic sources to replace widely used thermal light sources in a Michelson-interferometer based FTIR spectrometer, consisting of a moving mirror mounted on a mechanical delay line, as illustrated in figure 8(a). This is called single-comb FTIR spectroscopy. In contrast with an FTIR using an incoherent thermal light source, an FTIR spectrometer using a spatially coherent OPO frequency comb source dramatically reduces the measurement time owing to the high spectral brightness. An FTIR spectrometer based on an OPO frequency comb source supports the use of a multi-pass cell for enhancing detection sensitivity, and allows long-range open-path sensing and FTIR micro-spectroscopy. FTIR spectroscopy with an 
unstabilized OPO comb was first demonstrated by Tillman et al [76]. Adler et al reported the first implementation of high-resolution gas spectroscopy with a fully-stabilized OPO comb, achieving a spectral resolution of $0.0056 \mathrm{~cm}^{-1}$ and part-per-billion detection limits in $30 \mathrm{~s}$ of integration time [32]. Using intracavity spectroscopy, Haakestad et al demonstrated ultrasensitive detection of several different gas species in the spectral range $2.5-5 \mu \mathrm{m}$ by use of a broadband DRO comb, sharing coherence with its pump laser but not stabilized to an absolute reference [77].

A new approach for implementing frequency-comb spectroscopy is the dual-comb spectrometer, illustrated in figure 8(b), in which two asynchronous OPO combs with slightly different repetition rates (or mode spacings) are heterodyned before or after passing through a sample, yielding a down converted radio-frequency comb that contains information on the absorption experienced by the combs. The absence of moving parts in dual-comb spectrometers overcomes the speed and resolution limitations of a Michelsonbased spectrometer, and its resolution is only limited by the comb line-spacings. The recording time is the inverse of the difference in the repetition rates of combs. Dual-comb spectroscopy was initially demonstrated in mid-IR region by low power DFG combs [78, 79] or in near-IR region by modelocked laser frequency combs [80]. Zhang et al demonstrated the first OPO-based mid-infrared dual-comb spectroscopy by use of a unique scheme, in which two asynchronous mid-IR combs originated from one single OPO, and shared all the components for mid-IR generation and CEO-frequency detection [31, 81, 82]. Others have extended this approach by using two crystals in a shared OPO cavity produce two idler beams suitable for heterodyne detection of the dual-comb interferogram [83].

\subsection{Frequency metrology and calibration}

Femtosecond OPO frequency combs are ideal sources for frequency calibration in the near- to mid-IR, for applications in precision spectroscopy. Since stabilized OPO combs are not widely available, in all current demonstrations, frequencies of mid-IR lasers (e.g. quantum cascade lasers or single-frequency OPO sources) were calibrated either indirectly against a near-IR frequency comb via a nonlinear process [84, 85] or directly to a low power DFG comb [86]. Potentially, OPO frequency combs, which can provide higher power and direct coverage in the mid-IR region, will facilitate the implementation of precision mid-IR spectroscopy on a wider scale. In a preliminary study we used a $1.56 \mu \mathrm{m}$ femtosecond OPO comb to measure the absolute frequency of a Rb-referenced optical frequency standard, demonstrating performance fully matching that of a commercial fibre comb in the same near-infrared wavelength region [87]. In the near future we anticipate that femtosecond OPO combs will be suitable calibration sources for precision wavemeters and Fourier-transform spectrometers operating in the near- and mid-infrared.

\subsection{Quantum optics and optical computing}

Apart from using femtosecond degenerate OPOs as a tool for frequency-locked translation of the existing frequency comb sources to the longer wavelengths, they have rich quantum properties around the oscillation threshold. These properties are the consequence of the phase-sensitive nonlinear gain, which has been effectively used over the past few decades as a tool for generation of single-mode squeezed vacuum states in the cw regime [88]. It is also predicted that $\mathrm{cw}$ degenerate OPOs can potentially provide a qubit-like behaviour around the oscillation threshold [89]. In the frequency comb regime, non-classical behaviours of degenerate OPOs have been demonstrated below threshold [90], and they are utilized for realization of quantum networks [91] and all-optical quantum random number generation [33].

A network of time-multiplexed femtosecond degenerate OPOs have been utilized as a computing machine for Ising problems [34]. These problems map to many real-life combinatorial optimization problems, and belong to the nondeterministic polynomial-time (NP)-hard computational complexity class, for which no efficient classical and quantum algorithm exists. The performance of the OPO network in experiment and numerical simulations is promising as a new path for tackling these problems.

\subsection{Astrophotonics}

Laser frequency comb technology is emerging as the calibration source of choice for high resolution astronomical spectroscopy, however specific technical challenges stem from the difficulty of providing exceptionally wide comb-line spacings (6-32 GHz) across a three-octave wavelength range from $380 \mathrm{~nm}$ (Ca II H and $\mathrm{K}$ lines) to $2.4 \mu \mathrm{m}$ (CO band at $2.3 \mu \mathrm{m}$ ). Current solutions employ Fabry-Pérot filter cavities to increase the comb-line spacing, then amplify this filtered comb before launching into highly-nonlinear fibre to generate the required spectral broadening [92]. A technical issue with this approach (so far, only resolved by using multiple cascaded Fabry-Pérot filter cavities) is that nonlinear fibre-optic spectral broadening degrades side-mode suppression and causes side-mode amplitude asymmetry, which translates into wavelength calibration errors [93, 94]. The remarkably low thresholds of DRO combs offer an opportunity to realize high brightness, swept-wavelength calibration combs at multi-GHz repetition rates by direct pumping using multi-GHz Ti:sapphire lasers, such as the $10 \mathrm{GHz}$ system reported in 2008 by Bartels et al [60].

\section{Conclusions and perspectives}

In this article we have highlighted the advances made in the phase control of femtosecond OPOs, which led to the demonstration of OPO frequency combs. Such OPO combs share many common characteristics with their laser counterparts, however - as we have shown here-they also possess unique spectral and temporal properties that allow them to be 
exploited in applications beyond those addressable by currently available laser combs. For example, in the future we envisage low-threshold DRO frequency combs operating at high repetition rates, allowing them to be directly referenced to atomic transitions. The inherent quantum entanglement in such systems, and the potential to generate high repetition rate pulses containing only hundreds, rather than hundreds of thousands of modes, will make possible new continuousvariable approaches to quantum optics, acting as potential resources for quantum information processing. The extension of OPO combs to the spectral fingerprint region, for example using materials such as $\mathrm{CdSiP}_{2}$ [95], will also open new opportunities for dual-comb spectroscopy to become a powerful diagnostic tool by providing $\mathrm{kHz}$-rate ultra-high-resolution mid-IR spectroscopy for the analysis of systems characterized by complex and rapidly changing chemical signatures. Phase-stabilized OPO combs, based on the techniques described earlier, may also become useful as seed sources for mid-IR laser amplifiers, enabling CEP-stable $\mu \mathrm{J}$ pulses to be generated for high-harmonic generation, potentially at high repetition rates.

\section{Acknowledgments}

The results presented in this article would not have been possible without the contributions of a number of individuals, including: J Sun T I Ferreiro, K Balskus, B J S Gale, K L Vodopyanov, N C Leindecker, H Takada, and M Kakehata. Parts of this research were carried out under the METROCOMB project, which has received funding from the European Community's Seventh Framework Programme (FP7/ 2007-2013) under Grant Agreement no. 605057. The authors are also grateful for funding from the Science and Technology Facilities Council (STFC ST/L002140/1).

\section{References}

[1] Bartels A, Oates C W, Hollberg L and Diddams S A 2004 Stabilization of femtosecond laser frequency combs with subhertz residual linewidths Opt. Lett. 29 1081-3

[2] Schibli T R, Hartl I, Yost D C, Martin M J, Marcinkevičius A, Fermann M E and Ye J 2008 Optical frequency comb with submillihertz linewidth and more than $10 \mathrm{~W}$ average power Nat. Photonics 2 355-9

[3] Washburn B R, Diddams S A, Newbury N R, Nicholson J W, Yan M F and Jørgensen C G 2004 Phase-locked, erbiumfibre-laser-based frequency comb in the near infrared $O p t$. Lett. 29 250-2

[4] Jiang J, Mohr C, Bethge J, Fermann M E and Hartl I 2011 Fully stabilized, self-referenced thulium fibre frequency comb Conf. on Lasers and Electro-Optics Europe (E-CLEO) p PDB-1

[5] Tolstik N, Sorokin E and Sorokina I T 2013 Kerr-lens modelocked Cr:ZnS laser Opt. Lett. 38 299-301

[6] Cizmeciyan M N, Cankaya H, Kurt A and Sennaroglu A 2009 Kerr-lens mode-locked femtosecond $\mathrm{Cr}^{2+}: \mathrm{ZnSe}$ laser at $2420 \mathrm{~nm}$ Opt. Lett. 34 3056-8
[7] Gambetta A, Ramponi R and Marangoni M 2008 Mid-infrared optical combs from a compact amplified Er-doped fibre oscillator Opt. Lett. 33 2671-3

[8] Zhu F, Hundertmark H, Kolomenskii A A, Strohaber J, Holzwarth R and Schuessler H A 2013 High-power midinfrared frequency comb source based on a femtosecond $\mathrm{Er}$ : fibre oscillator Opt. Lett. 38 2360-2

[9] Edelstein D C, Wachman E S and Tang C L 1989 Broadly tunable high repetition rate femtosecond optical parametric oscillator Appl. Phys. Lett. 54 1728-30

[10] Udem T, Holzwarth R and Hänsch T W 2002 Optical frequency metrology Nature 416 233-7

[11] Gerginov V, Tanner C E, Diddams S A, Bartels A and Hollberg L 2005 High-resolution spectroscopy with a femtosecond laser frequency comb Opt. Lett. 30 1734-6

[12] Udem T, Reichert J, Holzwarth R and Hänsch T W 1999 Accurate measurement of large optical frequency differences with a mode-locked laser Opt. Lett. 24 881-3

[13] Chou C W, Hume D B, Koelemeij J C J, Wineland D J and Rosenband T 2010 Frequency comparison of two highaccuracy $\mathrm{Al}^{+}$optical clocks Phys. Rev. Lett. 104070802

[14] Yamada M 1994 Variation of intensity noise and frequency noise with the spontaneous emission factor in semiconductor lasers IEEE J. Quantum Electron. 30 1511-9

[15] Paschotta R 2010 Timing jitter and phase noise of mode-locked fibre lasers Opt. Express 18 5041-54

[16] Ferreiro T I, Sun J and Reid D T 2011 Frequency stability of a femtosecond optical parametric oscillator frequency comb Opt. Express 19 24159-64

[17] Tillman K A, Thapa R, Knabe K, Wu S, Lim J, Washburn B R and Corwin K L 2009 Stabilization of a selfreferenced, prism-based, Cr:forsterite laser frequency comb using an intracavity prism Appl. Opt. 48 6980-9

[18] Hartl I, Imeshev G, Fermann M E, Langrock C and Fejer M M 2005 Integrated self-referenced frequency-comb laser based on a combination of fibre and waveguide technology $O p t$. Express 13 6490-6

[19] Washburn B R, Diddams S A, Newbury N R, Nicholson J W, Yan M F and Jørgensen C G 2004 A phase locked, fibre laser-based frequency comb: limit on optical linewidth Conf. on Lasers and Electro-Optics (CLEO) paper CM03

[20] Sun J H, Gale B J S and Reid D T 2007 Composite frequency comb spanning 0.4-2.4 $\mu \mathrm{m}$ from a phase-controlled femtosecond $\mathrm{Ti}$ : sapphire laser and synchronously pumped optical parametric oscillator Opt. Lett. 32 1414-6

[21] Kobayashi Y and Torizuka K 2000 Measurement of the optical phase relation among subharmonic pulses in a femtosecond optical parametric oscillator Opt. Lett. 25 856-8

[22] Sun J, Gale B J S and Reid D T 2007 Testing the parametric energy conservation law in a femtosecond optical parametric oscillator Opt. Express 15 4378-84

[23] Ferreiro T I, Sun J and Reid D T 2010 Locking the carrierenvelope-offset frequency of an optical parametric oscillator without $f-2 f$ self-referencing Opt. Lett. 35 1668-70

[24] Wong S T, Vodopyanov K L and Byer R L 2010 Self-phaselocked divide-by-2 optical parametric oscillator as a broadband frequency comb source J. Opt. Soc. Am. B 27 876-82

[25] Adler F, Cossel K C, Thorpe M J, Hartl I, Fermann M E and Ye J 2009 Phase-stabilized, $1.5 \mathrm{~W}$ frequency comb at 2.8-4.8 $\mu \mathrm{m}$ Opt. Lett. 34 1330-2

[26] McCracken R A, Sun J, Leburn C G and Reid D T 2012 Broadband phase coherence between an ultrafast laser and an OPO using lock-to-zero CEO stabilization Opt. Express 20 16269-74

[27] Balskus K, Leitch S M, Zhang Z, McCracken R A and Reid D T $20151 \mathrm{GHz}$ harmonically pumped femtosecond optical parametric oscillator frequency comb Opt. Express 23 555-9 
[28] McCracken R A, Gianani I, Wyatt A S and Reid D T 2015 Multi-colour carrier-envelope-phase stabilization for highrepetition-rate multi-pulse coherent synthesis Opt. Lett. 40 $1208-11$

[29] Haakestad M W, Marandi A, Leindecker N and Vodopyanov K L 2013 Five-cycle pulses near $\lambda=3 \mu \mathrm{m}$ produced in a subharmonic optical parametric oscillator via fine dispersion management Laser Photonics Rev. 7 93-7

[30] Sun J and Reid D T 2009 Coherent ultrafast pulse synthesis between an optical parametric oscillator and a laser $O p t$. Lett. 34 854-6

[31] Zhang Z, Gardiner T and Reid D T 2013 Mid-infrared dualcomb spectroscopy with an optical parametric oscillator $O p t$. Lett. 38 3148-50

[32] Adler F, Masłowski P, Foltynowicz A, Cossel K C, Briles T C, Hartl I and Ye J 2010 Mid-infrared Fourier transform spectroscopy with a broadband frequency comb Opt. Express 18 21861-72

[33] Marandi A, Leindecker N C, Vodopyanov K L and Byer R L 2012 All-optical quantum random bit generation from intrinsically binary phase of parametric oscillators $O p t$ Express 20 19322-30

[34] Marandi A, Wang Z, Takata K, Byer R L and Yamamoto Y 2014 Network of time-multiplexed optical parametric oscillators as a coherent Ising machine Nat. Photonics 249 $1-6$

[35] Medeiros de Araújo R, Roslund J, Cai Y, Ferrini G, Fabre C and Treps N 2014 Full characterization of a highly multimode entangled state embedded in an optical frequency comb using pulse shaping Phys. Rev. A 89053828

[36] Smith R G, Geusic J E, Levinstein H J, Rubin J J, Singh S and Van Uitert L G 1968 Continuous optical parametric oscillation in $\mathrm{Ba}_{2} \mathrm{NaNb}_{5} \mathrm{O}_{15}$ Appl. Phys. Lett. 12 308-10

[37] Hänsch T W 1990 A proposed sub-femtosecond pulse synthesizer using separate phase-locked laser oscillators $O p t$. Commun. $8071-5$

[38] Shimoda K 1995 Theory and application of optical subharmonic oscillator Japan. J. Appl. Phys. 34 3566-9

[39] Kobayashi Y and Torizuka K 2001 Carrier-phase control among subharmonic pulses in a femtosecond optical parametric oscillator Opt. Lett. 26 1295-7

[40] Kobayashi Y, Takada H, Kakehata M and Torizuka K 2003 Optical phase locking among femtosecond subharmonic pulses Opt. Lett. 28 1377-9

[41] Kobayashi Y, Takada H, Kakehata M and Torizuka K 2003 Phase-coherent multicolour femtosecond pulse generation Appl. Phys. Lett. 83 839-41

[42] Jones D J, Diddams S A, Ranka J K, Stentz A, Windeler R S, Hall J L and Cundiff S T 2000 Carrier-envelope phase control of femtosecond mode-locked lasers and direct optical frequency synthesis Science $\mathbf{2 8 8}$ 635-9

[43] Apolonski A, Poppe A, Tempea G, Spielmann C, Udem T, Holzwarth R, Hänsch T W and Krausz F 2000 Controlling the phase evolution of few-cycle light pulses Phys. Rev. Lett. $85740-3$

[44] Sun J, Gale B J S and Reid D T 2007 Coherent synthesis using carrier-envelope phase-controlled pulses from a dual-colour femtosecond optical parametric oscillator Opt. Lett. 32 $1396-8$

[45] Kobayashi Y, Wei Z, Kakehata M, Takada H and Torizuka K 2003 Relative carrier-envelope-offset phase control between independent femtosecond light sources IEEE J. Sel. Top. Quantum Electron. 9 1011-7

[46] Yoshitomi D, Kobayashi Y, Kakehata M, Takada H and Torizuka K 2006 Synchronization of Ti: sapphire and Cr: forsterite mode-locked lasers with 100 attosecond precision by optical-phase stabilization Opt. Express 14 6359-65

[47] Cox J A, Putnam W P, Sell A, Leitenstorfer A and Kärtner F X 2012 Pulse synthesis in the single-cycle regime from independent mode-locked lasers using attosecond-precision feedback Opt. Lett. 37 3579-81

[48] Rossi G M, Cirmi G, Fang S, Chia S-H, Mücke O D, Kärtner F X, Manzoni C, Farinello P and Cerullo G 2014 Spectro-temporal characterization of all channels in a suboptical-cycle parametric waveform synthesizer Conf. on Lasers and Electro-Optics (CLEO) p SF1E.3

[49] Manzoni C, Huang S-W, Cirmi G, Farinello P, Moses J, Kärtner F X and Cerullo G 2012 Coherent synthesis of ultrabroadband optical parametric amplifiers Opt. Lett. 37 $1880-2$

[50] Yoshitomi D, Zhou X, Kobayashi Y, Takada H and Torizuka K 2010 Long-term stable passive synchronization of $50 \mu \mathrm{J}$ femtosecond $\mathrm{Yb}$-doped fibre chirped-pulse amplifier with a mode-locked Ti:sapphire laser Opt. Express 18 26027-36

[51] Ferreiro T I 2013 Development and characterisation of a nearinfrared femtosecond optical parametric oscillator frequency comb PhD Thesis Heriot-Watt University

[52] Allan D W 1966 Statistics of atomic frequency standards Proc. IEEE 54 221-30

[53] Sun J, Gale B J S and Reid D T 2006 Dual-colour operation of a femtosecond optical parametric oscillator exhibiting stable relative carrier-envelope phase-slip frequencies Opt. Lett. 31 2021-3

[54] Bartels A, Newbury N R, Thomann I, Hollberg L and Diddams S A 2004 Broadband phase-coherent optical frequency synthesis with actively linked Ti:sapphire and $\mathrm{Cr}$ : forsterite femtosecond lasers Opt. Lett. 29 403-5

[55] Kobayashi Y, Yoshitomi D, Kakehata M, Takada H and Torizuka K 2005 Long-term optical phase locking between femtosecond Ti:sapphire and Cr:forsterite lasers Opt. Lett. 30 2496-8

[56] Reid D T, McGowan C, Sleat W, Ebrahimzadeh M and Sibbett W 1997 Compact, efficient $344 \mathrm{MHz}$ repetition-rate femtosecond optical parametric oscillator Opt. Lett. 22 $525-7$

[57] Ruffing B, Nebel A and Wallenstein R 1998 All-solid-state cw mode-locked picosecond $\mathrm{KTiOAsO}_{4}$ (KTA) optical parametric oscillator Appl. Phys. B 67 537-44

[58] Jiang J and Hasama T 2002 Harmonic repetition-rate femtosecond optical parametric oscillator Appl. Phys. B $\mathbf{7 4}$ 313-7

[59] Kokabee O, Esteban-Martin A and Ebrahim-Zadeh M 2009 Extended-cavity, tunable, GHz-repetition-rate femtosecond optical parametric oscillator pumped at $76 \mathrm{MHz} O p t$. Express 17 15635-40

[60] Bartels A, Heinecke D and Diddams S A 2008 Passively modelocked $10 \mathrm{GHz}$ femtosecond Ti:sapphire laser Opt. Lett. 33 1905-190

[61] Byer R L 1997 Parametric oscillators and nonlinear materials Nonlinear Optics ed P G Harper and B S Wherrett (New York: Academic) pp 47-160

[62] Gebs R, Dekorsy T, Diddams S A and Bartels A $20081 \mathrm{GHz}$ repetition rate femtosecond $\mathrm{OPO}$ with stabilized offset between signal and idler frequency combs Opt. Express 16 5397-405

[63] Reid D T 2011 Ultra-broadband pulse evolution in optical parametric oscillators Opt. Express 19 17979-84

[64] Wolf S J, Phillips C R, Marandi A, Vodopyanov K L, Fejer M M and Byer R L 2013 Dynamical behavior of an ultrafast OPO near degeneracy Conf. on Lasers and ElectroOptics (CLEO) p CW1B.1

[65] Marandi A, Leindecker N C, Pervak V, Byer R L and Vodopyanov K L 2012 Coherence properties of a broadband femtosecond mid-IR optical parametric oscillator operating at degeneracy Opt. Express 20 7255-62

[66] Herr T, Hartinger K, Riemensberger J, Wang C Y, Gavartin E, Holzwarth R, Gorodetsky M L and Kippenberg T J 2012 
Universal formation dynamics and noise of Kerr-frequency combs in microresonators Nat. Photonics 6 480-7

[67] Lee K F, Jiang J, Mohr C, Bethge J, Fermann M E, Leindecker N, Vodopyanov K L, Schunemann P G and Hartl I 2013 Carrier envelope offset frequency of a doubly resonant, nondegenerate, mid-infrared GaAs optical parametric oscillator Opt. Lett. 38 1191-3

[68] Wong S T, Plettner T, Vodopyanov K L, Urbanek K, Digonnet M and Byer R L 2008 Self-phase-locked degenerate femtosecond optical parametric oscillator $\mathrm{Opt}$. Lett. 33 1896-8

[69] Ramaiah-Badarla V, Esteban-Martin A and Ebrahim-Zadeh M 2013 Self-phase-locked degenerate femtosecond optical parametric oscillator based on $\mathrm{BiB}_{3} \mathrm{O}_{6}$ Laser Photonics Rev. $71-6$

[70] Rudy C W, Marandi A, Ingold K A, Wolf S J, Vodopyanov K L, Byer R L, Yang L, Wan P and Liu J 2012 Sub-50 fs pulses around $2070 \mathrm{~nm}$ from a synchronously-pumped, degenerate OPO Opt. Express 20 27589-95

[71] Leindecker N, Marandi A, Byer R L, Vodopyanov K L, Jiang J, Hartl I, Fermann M and Schunemann P G 2012 Octave-spanning ultrafast $\mathrm{OPO}$ with $2.6-6.1 \mu \mathrm{m}$ instantaneous bandwidth pumped by femtosecond Tm-fibre laser Opt. Express 20 7046-53

[72] Marandi A, Rudy C W, Plotnichenko V G, Dianov E M, Vodopyanov K L and Byer R L 2012 Mid-infrared supercontinuum generation in tapered chalcogenide fibre for producing octave-spanning frequency comb around $3 \mu \mathrm{m}$ Opt. Express 20 24218-25

[73] Lee K F, Mohr C, Leindecker N, Vodopyanov K L, Schunemann P G, Hartl I and Fermann M E 2013 Mid-IR frequency comb with sub-hertz residual linewidth from a doubly-resonant OPGaAs OPO Conf. on Lasers and Electro-Optics Pacific Rim (CLEO-PR) pp TuC1-4

[74] Bosenberg W R, Drobshoff A, Alexander J I, Myers L E and Byer R L 1996 93\% pump depletion, 3.5 W continuouswave, singly resonant optical parametric oscillator Opt. Lett. 21 1336-8

[75] Ingold K A, Marandi A, Rudy C W and Byer R L 2014 $2.09 \mu \mathrm{m}$ degenerate femtosecond OPO with over $60 \%$ conversion efficiency and $0.6 \mathrm{~W}$ output Conf. on Lasers and Electro-Optics (CLEO) p SM2I.4

[76] Tillman K A, Maier R R J, Reid D T and McNaghten E D 2004 Mid-infrared absorption spectroscopy across a $14.4 \mathrm{THz}$ spectral range using a broadband femtosecond optical parametric oscillator Appl. Phys. Lett. 85 3366-8

[77] Haakestad M W, Lamour T P, Leindecker N, Marandi A and Vodopyanov K L 2013 Intracavity trace molecular detection with a broadband mid-IR frequency comb source $J$. Opt. Soc. Am. B 30 631-40

[78] Schliesser A, Brehm M, Keilmann F and van der Weide D W 2005 Frequency-comb infrared spectrometer for rapid, remote chemical sensing Opt. Express 13 9029-38

[79] Baumann E, Giorgetta F R, Swann W C, Zolot A M, Coddington I and Newbury N R 2011 Spectroscopy of the methane $\mathrm{v} 3$ band with an accurate midinfrared coherent dual-comb spectrometer Phys. Rev. A 84 062513
[80] Coddington I, Swann W C and Newbury N R 2008 Coherent multiheterodyne spectroscopy using stabilized optical frequency combs Phys. Rev. Lett. 100013902

[81] Zhang Z, Gu C, Sun J, Wang C, Gardiner T and Reid D T 2012 Asynchronous midinfrared ultrafast optical parametric oscillator for dual-comb spectroscopy Opt. Lett. 37 187-9

[82] Zhang Z, Fang X, Gardiner T and Reid D T 2013 High-power asynchronous midinfrared optical parametric oscillator frequency combs Opt. Lett. 38 2077-9

[83] In Y J, Cristescu S M, Harren F J M and Mandon J 2014 Twocrystal mid-infrared optical parametric oscillator for absorption and dispersion dual-comb spectroscopy Opt. Lett. 39 3270-3

[84] Knabe K, Williams P A, Giorgetta F R, Radunsky M B, Armacost C M, Crivello S and Newbury N R 2013 Absolute spectroscopy of $\mathrm{N}_{2} \mathrm{O}$ near $4.5 \mu \mathrm{m}$ with a comb-calibrated, frequency-swept quantum cascade laser spectrometer $O p t$. Express 21 1020-9

[85] Peltola J, Vainio M, Fordell T, Hieta T, Merimaa M and Halonen L 2014 Frequency-comb-referenced mid-infrared source for high-precision spectroscopy Opt. Express 22 32429-39

[86] Gambetta A, Cassinerio M, Coluccelli N, Fasci E, Castrillo A, Giangrani L, Gatti D, Marangoni M, Laporta P and Galzerano G 2015 Direct phase-locking of a $8.6 \mu \mathrm{m}$ quantum cascade laser to a mid-IR optical frequency comb: application to precision spectroscopy of $\mathrm{N}_{2} \mathrm{O}$ Opt. Lett. 40 304-7

[87] Schilt S et al 2015 Noise characterization and optical frequency measurement with an optical parametric oscillator frequency comb 8th Symp. on Frequency Standards and Metrology (Potsdam, Germany) submitted

[88] Wu L-A, Kimble H J, Hall J L and Wu H 1986 Generation of squeezed states by parametric down conversion Phys. Rev. Lett. 57 2520-3

[89] Wolinsky M and Carmichael H J 1988 Quantum noise in the parametric oscillator: from squeezed states to coherent-state superpositions Phys. Rev. Lett. 60 1836-9

[90] Pinel O, Jian P, de Araújo R M, Feng J, Chalopin B, Fabre C and Treps N 2012 Generation and characterization of multimode quantum frequency combs Phys. Rev. Lett. 108083601

[91] Roslund J, Medeiros de Araújo R, Jiang S, Fabre C and Treps N 2013 Wavelength-multiplexed quantum networks with ultrafast frequency combs Nat. Photonics 8 109-12

[92] Mégevand D et al 2012 ESPRESSO, the ultimate rocky exoplanets hunter for the VLT Proc. SPIE 8446 84461R

[93] Wilken T et al 2012 A spectrograph for exoplanet observations calibrated at the centimetre-per-second level Nature $\mathbf{4 8 5}$ 611-4

[94] Quinlan F, Ycas G, Osterman S and Diddams S A 2010 A $12.5 \mathrm{GHz}$-spaced optical frequency comb spanning $>400 \mathrm{~nm}$ for near-infrared astronomical spectrograph calibration Rev. Sci. Instrum. 81063105

[95] Zhang Z, Reid D T, Kumar S C, Ebrahim-Zadeh M, Schunemann P G, Zawilski K T and Howle C R 2013 Femtosecond-laser pumped $\mathrm{CdSiP}_{2}$ optical parametric oscillator producing $100 \mathrm{MHz}$ pulses centred at $6.2 \mu \mathrm{m} \mathrm{Opt}$. Lett. 38 5110-3 